\title{
Validity of three screening questions (3Q/TMD) in relation to the DC/TMD
}

\author{
A. LÖVGREN*,C. M. VISSCHER ${ }^{\dagger}$, B. HÄGGMAN-HENRIKSON**, F. LOBBEZOO ${ }^{\dagger}$, \\ S. MARKLUND* \& A. W ÄNMAN* *Department of Clinical Oral Physiology, Faculty of Medicine, University of \\ Umeå, Umeå, Sweden, †Department of Oral Kinesiology, Academic Centre for Dentistry Amsterdam (ACTA), University of Amsterdam and \\ VU University Amsterdam, MOVE Research Institute Amsterdam, Amsterdam. The Netherlands and "Department of Orofacial Pain and \\ Jaw Function, Faculty of Odontology, Malmö University, Malmö, Sweden
}

SUMMARY Temporomandibular disorders (TMD) are common but seem to be largely undetected within general dental care. To improve dentists' awareness of these symptoms, three screening questions (3Q/TMD) have been introduced. Our aim was to validate $3 Q / T M D$ in relation to the diagnostic criteria for TMD (DC/TMD), while taking into account the severity level of the symptoms. The study population consisted of 7831 individuals 20-69 years old, who had their routine dental check-up at the Public Dental Health Service in Västerbotten, Sweden. All patients answered a health declaration, including the $3 Q /$ TMD regarding frequent temporomandibular pain, pain on movement and catching/locking of the jaw. All 3Q-positives (at least one affirmative) were invited for examination in randomised order. For each 3Q-positive, a matched 3Q-negative was invited. In total, 152 3Q-positives and 148 3Qnegatives participated. At examination, participants answered 3Q/TMD a second time, before they were examined and diagnosed according to DC/TMD. To determine symptom's severity, the Graded Chronic Pain Scale and Jaw Functional Limitation Scale-20 (JFLS-20) were used. In total, $74 \%$ of $3 Q$-positives and $16 \%$ of $3 Q$ negatives met the criteria for DC/TMD pain or dysfunction (disc displacements with reduction and degenerative joint disorder were excluded). Fifty-five per cent of 3Q-positives had a TMD diagnosis and CPI score $\geq 3$ or a JFLS-20 score $\geq 5$, compared to $4 \%$ of $3 \mathrm{Q}$-negatives. The results show that the $3 Q / T M D$ is an applicable, cost-effective and valid tool for screening a general adult population to recognise patients in need of further TMD examination and management.

KEYWORDS: temporomandibular disorders, oro-facial pain/TMD, diagnostic test, routine, epidemiology, health services research, practice guidelines as topic

Accepted for publication 7 August 2016

\section{Background}

Temporomandibular disorders (TMD) is a term commonly used to group conditions in the masticatory muscles and the temporomandibular joint (TMJ), impaired movement capacity of the mandible, and TMJ symptoms such as clicking, grating and locking of the jaw (1). The prevalence of TMD is highly dependent on included signs and symptoms, and their occurrence in terms of frequency and duration. It has been reported that temporomandibular pain affects approximately $10 \%$ of the adult population (2) and is considered to be the most common chronic oro-facial pain condition $(3,4)$. If all symptoms involving TMD are included, the prevalence increases up to $50 \%$ of the adult population (5).

Despite the fact that TMD pose a negative impact on daily life (6), these conditions seem to be largely undetected or neglected in dental health care. This is illustrated by a discrepancy between estimated 
treatment need and traceable, performed treatment (7-9). The reason for a substantial difference between estimated treatment need and actual treatment carried out is unknown and constitutes a gap of knowledge within dentistry.

To improve dentists' awareness of symptoms indicative of TMD among their patients and to improve the quality of the dental care for patients with TMD, three mandatory screening questions (3Q/ TMD) were introduced in 2010 in the dental digital record system in the county of Västerbotten, Sweden (10). The aim of the screening questions was to identify patients with more severe TMD at regular dental check-ups. Two questions addressed frequent pain in the temporomandibular region, and one question addressed frequent locking and catching of the TMJ. The questions on pain have been validated in relation to the Research Diagnostic criteria (RDC/TMD) in adolescents (11) but not in adults, while the validity of the third question has not yet been established.

Other screening questions aimed at detecting patients with possible TMD are available. One of these, the TMD Pain screener, focusing on pain within the last 30 days, is part of the symptom questionnaire for the Diagnostic Criteria for TMD (DC/TMD) (12). Since 2014, the (DC/TMD) is recommended for use in both clinical and research settings (13). Axis I is constructed to arrive at a diagnosis related to TMD pain conditions (myalgia, arthralgia and headaches attributed to TMD), as well as to a TMD diagnosis related to intra-articular disorders (disc displacements and degenerative joint disease).

The aim of this study was to validate the 3Q/TMD in adults in relation to the DC/TMD, characteristic pain intensity (CPI) and functional limitations. Our hypothesis was that $3 \mathrm{Q} / \mathrm{TMD}$ is an applicable screening system for identifying adults with a more severe TMD diagnosis and functional impairment of the masticatory system.

\section{Methods}

\section{Study population}

The study population consisted of 7831 individuals, who attended the Public Dental Health Service (PDHS) in Västerbotten in 2014 for their routine dental check-ups. All patients answered a health declaration including the three screening questions (3Q/TMD) at their appointment (T1) and, based on their answers, were classified as 3Q-positive or 3Qnegative. The 3Q-positives $(n=524)$ constituted those who gave an affirmative answer to one or more of the questions. The 3Q-negatives $(n=7279)$ gave negative answers to all three questions and were stratified in five 10-year age clusters (20-29; 30-39; 40-49; 50-59; 60-69 years). All 3Q-positives (at least one affirmative) were invited for examination in randomised order (SPSS v.22; IBM, New York, USA, random numbers). For each 3Q-positive, an age cluster- and gender-matched 3Q-negative was invited. Both 3Q-positives and 3Q-negatives were invited by letter, with brief information about the study and an offer to participate in the clinical examination free of charge. They were contacted by phone approximately one week later, and if they accepted to participate, they were scheduled for the clinical examination. In total, 335 3Q-positive and 342 3Q-negative patients were invited, and of these 152 3Q-positives and 148 3Q-negatives participated. Based on a power analysis using a sensitivity of $0 \cdot 8$ and specificity of $0 \cdot 8$, an estimated prevalence of $50 \%$ and a precision of $0 \cdot 1$, the total sample size was 250 .

Participants received remuneration equivalent to 20 euros to cover travelling expenses.

\section{Reference standard}

The clinical examinations were carried out by a single, blinded examiner, who had been formally two-day trained and calibrated according to the DC/TMD course (14).

The examination procedure followed the DC/TMD protocol including the DC/TMD Symptom Questionnaire (DC/TMD-SQ). For this study, the Jaw function limitation scale-20 (JFLS-20) (15) and the Graded Chronic Pain Scale (GCPS) (16) from AXIS II were used in the analyses. All participants were diagnosed according to the DC/TMD (13). Characteristic pain intensity in the jaw/face region was rated on a numerical rating scale $(0-10)$ for current pain, worst pain in the past month, and average pain the past month (17), and the mean value of these three values was calculated. The functional status of the masticatory system was assessed by JFLS-20 that includes 20 items related to jaw function rated on a $0-10$ scale (i.e. no limitation to severe limitation). 


\section{Q/TMD}

The three questions are formulated as follows:

1 Q1: 'Do you have pain in your temple, face, jaw or jaw joint once a week or more?'

2 Q2: 'Do you have pain once a week or more when you open your mouth or chew?'

3 Q3: 'Does your jaw lock or become stuck once a week or more?'

On the day of the examination, all participants completed the $3 \mathrm{Q} / \mathrm{TMD}$ a second time (T2), before the clinical examination and these answers were used in the analysis, as they most closely matched the current complaints of the participant.

A written consent was obtained from all participants, and the STROBE statement was followed.

\section{Statistical methods}

Differences in age, gender and severity of symptoms within and between groups were analysed with chisquare and Mann-Whitney U-tests. Sensitivity, specificity, positive predictive values (PPV) and negative predictive values (NPV) were calculated. Confidence intervals $(95 \%-\mathrm{CI})$ were calculated by the Wilson 'score' method (18). As reference standard, DC/TMD pain (arthralgia or myalgia) was used for Q1 and Q2, respectively, and combined. For Q3, DC/TMD dysfunction (disc displacement with reduction and intermittent locking, disc displacement without reduction with and without limited opening or subluxation) was used. As disc displacement with reduction and degenerative joint disorder primarily relate to joint sounds, and may not include functional limitations such as jaw locking or restricted movements, these DC/TMD diagnoses were not included as reference standard. The predictive values were calculated based on the following assumed prevalence in the general population: TMD pain 10\%; TMD dysfunction 5\% (5), and in a specialist setting: TMD pain 50\%, TMD dysfunction $10 \%$. For the CPI, 0-2 was considered as low intensity, and $\geq 3$ as moderate- to high-intensity level. For the JFLS-20, participants who stated 5 or more on the 0-10 scale of any of the 20 questions were appointed significant limitation; the remaining participants were classified as having no/low impairment. In the analysis, 'more severe TMD' was defined as participants with a DC/TMD diagnosis combined with moderate or high pain intensity (CPI $\geq 3$ ) or significant limitation (JFLS-20 $\geq 5$ ). The statistical analysis was carried out with SPSS version 22 , and a $P$-value $<0.05$ was considered statistically significant.

\section{Results}

In total, 300 participants (202 women and 98 men), aged between 20 and 69 years (mean age 3Q-positives 38.3 years s.d.: 13.9; mean age 3Q-negatives 39.1 years s.d. 13.6) were examined. The average time between randomisation (T1) and examination (T2) was 16.3 weeks (s.d. 7.3) among 3Q-positives and 16.2 weeks (s.d. 6.7) among 3Q-negatives. At the day of examination (T2), 37 (24\%) of the original 3Q-positive participants no longer reported an affirmative answer to at least one of the 3Q/TMD and were subsequently reclassified as 3Q-negatives. Furthermore, 25 (17\%) of the original 3Q-negative participants reported at least one affirmative answer to the $3 \mathrm{Q} / \mathrm{TMD}$ on the day of the examination and were re-classified as $3 \mathrm{Q}$ positives (Fig. 1). The gender distribution among 3Qpositives was 101 women and 39 men (mean age 36.9 years s.d. 13.7), and among 3Q-negatives 101 women and 59 men (mean age $40 \cdot 2$ years s.d. 13.6). There were no significant differences in age (T1; $P=0.997, \mathrm{~T} 2 ; P=0.144)$ or gender distribution (T2; $P=0.097)$ between these groups.

The distribution of diagnoses based on the clinical examination among 3Q-positives and 3Q-negatives are presented in Fig. 2. In total, 74\% of the 3Q-positives and $16 \%$ of 3Q-negatives met the criteria for the DC/TMD diagnoses included in the main analysis.

\section{$\begin{array}{ll}\mathrm{T} 1 & \mathrm{~T} 2\end{array}$}

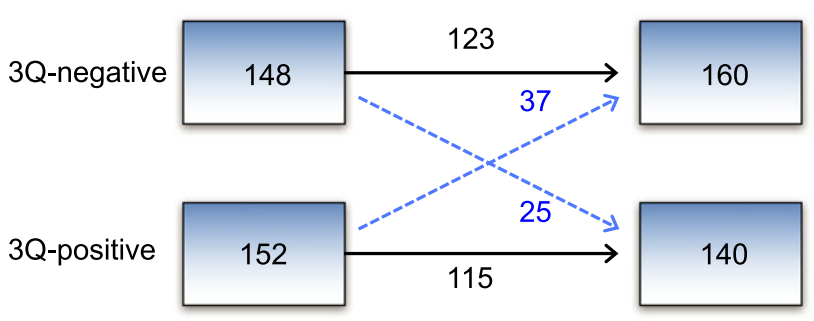

Fig. 1. Flow chart of distribution of 3Q-positives and 3Q-negatives at the different time-points, based on the answers to $3 \mathrm{Q} /$ TMD at regular dental check-up, which served as the basis for randomisation ( $\mathrm{Tl}$ ), and the subjects' answer to the same questions at the time of the blinded research examination (T2). The dashed lines indicate individuals re-classified between these time-points due to shift in answers. In total, 300 individuals were enrolled for the study. 


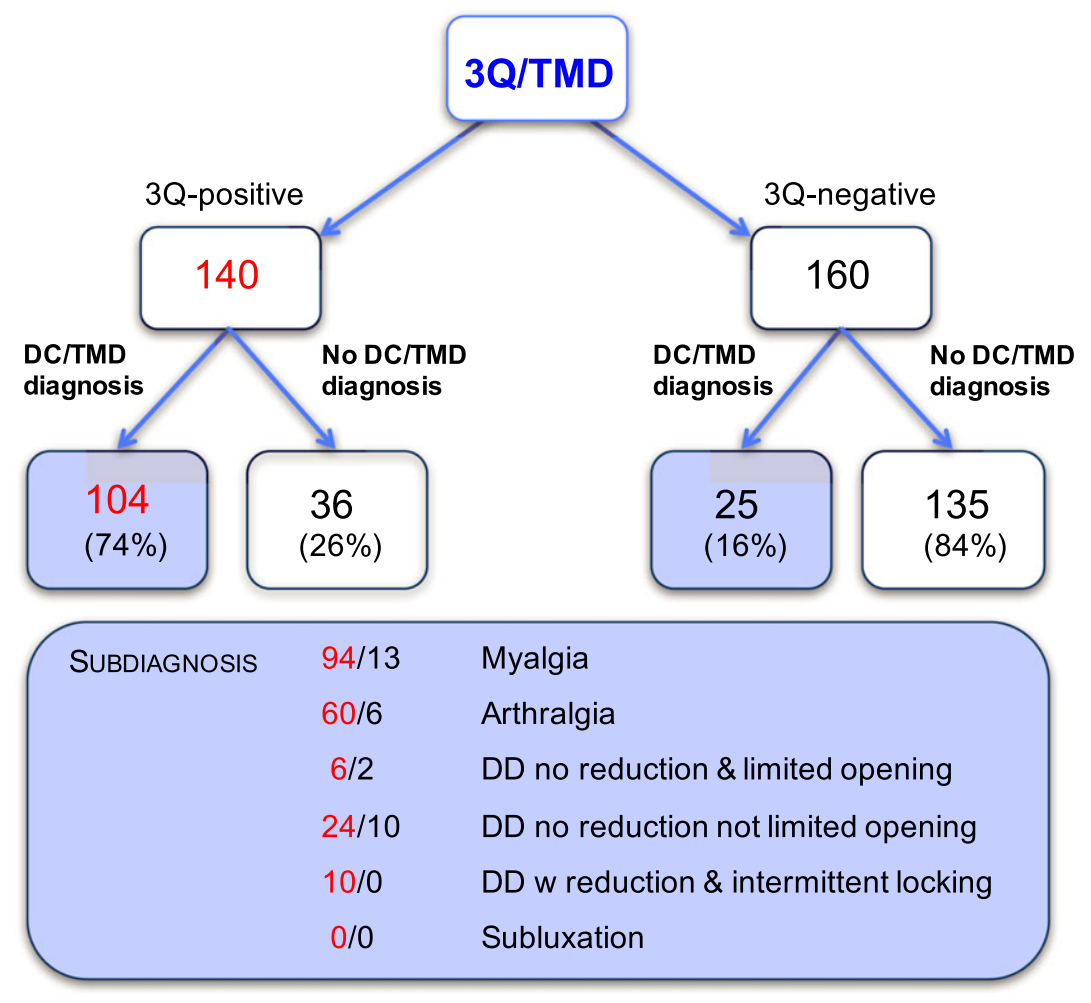

Fig. 2. The distribution of diagnoses used in the analysis among 3Qpositives and 3Q-negatives based on answers to the 3Q/TMD (disc displacement, DD with reduction and degenerative joint disorder excluded).
3Q-positives reported significantly higher pain intensity levels (median CPI: 3.3 , range: $0-8 \cdot 7 ; P<0.001$ ), and more jaw functional limitations (median JFLS: 11 , range $0-117 ; P<0.001)$ compared to 3Q-negatives (median CPI: 0, range: 0-6; median JFLS: 0, range: $0-72$ ). Thus, $55 \%$ of the 3Q-positives received a diagnosis combined with a CPI $\geq 3$ or a score $\geq 5$ on JFLS-20 compared to $4 \%$ of the 3Q-negatives $\left(\chi^{2}\right.$ value 97.998; $P<0.001)$. The prevalence of disc displacement with reduction or degenerative joint disorder was $32 \%$ and $27 \%$ among 3Q-positives compared to $34 \%$ and $21 \%$ among 3Q-negatives, respectively. The sensitivities and specificities and related positive and negative predictive values for affirmative answers to $3 \mathrm{Q} / \mathrm{TMD}$ are presented in Table 1 . When applied to a general practice sample, 3Q/TMD, separately or in combinations, showed high NPV (0.92-0.99). The corresponding PPV was highest for a TMD pain diagnosis when both Q1 and Q2 were affirmative (0.59;0.55). Two or more affirmatives were related to the highest PPV $(0 \cdot 65 ; 0.51)$ for the reference diagnoses. The PPV was high $(0 \cdot 93 ; 0.92)$ for Q1 and Q2 in relation to a TMD pain diagnosis when applied to the expected prevalence in a specialist clinic. Two or more affirmatives also showed high PPV $(0 \cdot 94 ; 0 \cdot 90)$ for any of the reference diagnoses in such a setting.

\section{Discussion}

The main finding of the present study was that the two screening questions related to frequent pain (Q1, Q2) showed substantial validity in relation to DC/ TMD pain (myalgia/arthralgia) among adults. The question related to frequent impairment of jaw function (Q3) showed fair-to-moderate validity to DC/ TMD dysfunction.

In daily general dental practice, patients with TMD seem to be largely disregarded $(7,9)$. Unidentified disorders may cause unnecessary suffering and may affect other treatments negatively. A perfect screening test should be easy and identify all true positives and negatives correctly. Screening tests related to nonmortal diseases of low prevalence in the population require a high specificity (few false positives) (19). The tested 3Q/TMD showed high specificity especially if two or more of the questions were answered affirmatively, which supports their use in the general dental practice. When applying the 3Q/TMD, most patients that are not referred for further diagnostics will indeed have no TMD (high specificity). Patients that are referred, however, might show to have a TMD, although also patients with other oro-facial complaints such as, for example patients with 
Table 1. Sensitivity, specificity, positive predictive values (PPV) and negative predictive values (NPV) for Q1, Q2 in relation to DC/ TMD pain; Q3 to DC/TMD dysfunction; and one or more affirmative to any DC/TMD diagnosis

\begin{tabular}{|c|c|c|c|c|c|c|}
\hline & \multirow[b]{2}{*}{ Sensitivity } & \multirow[b]{2}{*}{ Specificity } & \multicolumn{2}{|l|}{ General practice $^{\dagger}$} & \multicolumn{2}{|l|}{ Specialist practice ${ }^{\ddagger}$} \\
\hline & & & PPV & NPV & PPV & NPV \\
\hline Q1 & $0.78(0.70-0 \cdot 84)$ & $0.89(0.83-0.93)$ & $0.44(0.39-0.50)$ & $0.97(0.94-0.98)$ & $0.88(0.82-0.93)$ & $0.80(0.78-0.82)$ \\
\hline Q2 & $0.56(0.49-0.63)$ & $0.94(0.88-0.98)$ & $0.51(0.45-0.57)$ & $0.95(0.92-0.96)$ & $0.90(0.84-0.95)$ & $0.68(0.66-0.70)$ \\
\hline Q1 or Q2 & $0.82(0.74-0.88)$ & $0.87(0.81-0.91)$ & $0.41(0.36-0.47)$ & $0.98(0.95-0.99)$ & $0.86(0 \cdot 80-0.91)$ & $0.83(0.81-0.85)$ \\
\hline Q1 and Q2 & $0.52(0.45-0.59)$ & $0.96(0.90-1 \cdot 0)$ & $0.59(0.53-0.65)$ & $0.95(0.92-0.96)$ & $0.93(0.87-0.98)$ & $0.67(0.65-0.69)$ \\
\hline Q3 & $0.45(0.38-0.52)$ & $0.86(0 \cdot 80-0 \cdot 90)$ & $0 \cdot 15(0 \cdot 10-0 \cdot 21)$ & $0.97(0.94-0.98)$ & $0.26(0.21-0.32)$ & $0.93(0.91-0.95)$ \\
\hline$\geq 1$ affirmative & $0.81(0.73-0.87)$ & $0.79(0.73-0.83)$ & $0.30(0 \cdot 25-0 \cdot 36)$ & $0.97(0.94-0.98)$ & $0.79(0.73-0.84)$ & $0.81(0.79-0.83)$ \\
\hline$\geq 2$ affirmative & $0.50(0.43-0.57)$ & $0.97(0.91-1 \cdot 0)$ & $0.65(0.59-0.70)$ & $0.95(0.92-0.96)$ & $0.94(0.88-0.99)$ & $0.66(0.64-0.68)$ \\
\hline 3 affirmative & $0.21(0 \cdot 15-0.29)$ & $0.99(0.93-1.0)$ & $0 \cdot 70(0 \cdot 64-0 \cdot 75)$ & $0.92(0.90-0.94)$ & $0.96(0 \cdot 90-1 \cdot 0)$ & $0.56(0.54-0.58)$ \\
\hline \multicolumn{7}{|l|}{ More severe TMD } \\
\hline Q1 & $0.87(0.79-0.93)$ & $0.82(0.76-0.86)$ & $0.35(0.30-0.41)$ & $0.98(0.95-0.99)$ & $0.83(0.77-0.88)$ & $0.86(0.84-0.88)$ \\
\hline Q2 & $0.70(0.63-0.76)$ & $0.92(0.86-0.96)$ & $0.49(0.43-0.55)$ & $0.97(0.94-0.98)$ & $0.90(0.84-0.95)$ & $0.75(0.73-0.77)$ \\
\hline Q1 or Q2 & $0.90(0.82-0.96)$ & $0.79(0.73-0.83)$ & $0.32(0 \cdot 27-0 \cdot 38)$ & $0.99(0.96-1.0)$ & $0.81(0.75-0.86)$ & $0.89(0.87-0.91)$ \\
\hline Q1 and Q2 & $0.67(0.60-0.73)$ & $0.94(0.88-0.98)$ & $0.55(0.49-0.61)$ & $0.96(0.93-0.97)$ & $0.92(0.86-0.97)$ & $0.74(0.72-0.76)$ \\
\hline Q3 & $0.55(0.48-0.62)$ & $0.85(0.79-0.89)$ & $0 \cdot 16(0 \cdot 11-0 \cdot 22)$ & $0.97(0.94-0.98)$ & $0.29(0.24-0.35)$ & $0.95(0.92-0.96)$ \\
\hline$\geq 1$ affirmative & $0.93(0.85-0.99)$ & $0.71(0.66-0.75)$ & $0 \cdot 26(0 \cdot 21-0 \cdot 32)$ & $0.99(0.96-1 \cdot 0)$ & $0.76(0.70-0.81)$ & $0.91(0.89-0.93)$ \\
\hline$\geq 2$ affirmative & $0.66(0.59-0.72)$ & $0.93(0.87-0.97)$ & $0.51(0.45-0.57)$ & $0.96(0.93-0.97)$ & $0.90(0.84-0.95)$ & $0.73(0.71-0.75)$ \\
\hline 3 affirmative & $0.29(0.23-0.36)$ & $0.98(0.92-1.0)$ & $0.62(0.56-0.67)$ & $0.93(0.90-0.95)$ & $0.94(0.88-0.99)$ & $0.58(0.56-0.60)$ \\
\hline
\end{tabular}

DC/TMD pain: myalgia or arthralgia.

DC/TMD dysfunction: disc displacement with reduction and intermittent locking, disc displacement without reduction with and without limited opening or subluxation.

Bold figures indicate optimal use in clinical practice.

A $95 \%$ confidence interval is given within parenthesis.

${ }^{\dagger}$ With estimated prevalence for general practice $10 \%$ for TMD pain diagnosis and $5 \%$ for dysfunction.

*With estimated prevalence for specialist practice $50 \%$ for TMD pain diagnosis and $10 \%$ for dysfunction.

toothache, respond positively to the $3 \mathrm{Q} / \mathrm{TMD}$ (as illustrated by the moderate sensitivity). This can probably relate to the broad nature of the questions, which means that also individuals not affected by TMD will provide a positive answer. This could be regarded as a limitation of the screening method in relation to TMD. On the other hand, as these individuals do report an oro-facial pain complaint they will probably benefit from a further examination to determine the cause.

Translating these group estimates of validity (i.e. specificity and sensitivity) to individual prediction of the chance that a screening outcome predicts the presence of a TMD illustrates that when applied in the general practice the negative predictive value (NPV) is excellent, while the positive predictive value (PPV) is lower. Extrapolating these figures to a specialised clinic, where the prevalence of TMD pain and dysfunction are higher, the PPV increases at the expense of the NPV. The predictive values are highly dependent on the prevalence of the condition, which is illustrated in Table 1. Further studies are needed to determine the validity in a specialised clinic setting.

The sensitivity and specificity for the two pain questions in the present study were lower than those reported in a similar study design on adolescents ( 11 , 20 ). This finding was not surprising since the previous studies used the RDC/TMD while we used the DC/ TMD as the reference standard, which is assumed to have a better validity for the recognition of TMD (13). One important difference between the 3Q/TMD and the DC/TMD criteria is the difference in the frequency of symptoms. Based on a critical review (21) that advocated that a time frame of TMD symptoms once a week or more is more clinically relevant and reliable, the screening questions 3Q/TMD focus on weekly symptoms, while the DC/TMD asks for symptoms within the last 30 days. Participants, who received a DC/TMD pain diagnosis (myalgia or arthralgia) in spite of a negative answer to Q1, might thus have experienced pain during the past month, but without weekly symptoms. 
The third question in the 3Q/TMD was constructed to capture TMJ conditions that hamper jaw function significantly and may indicate demand for treatment. Disc displacements with reduction and degenerative joint disorder were therefore not included as part of the reference standard. The outcomes of our study showed that these subtypes of TMD were equally present among 3Q-positives and 3Q-negatives, which confirms the intention to screen for functional disturbances other than joint sounds. Disc displacements without reduction and disc displacements with reduction and intermittent locking were more prevalent among the 3Q-positives. Still, almost $60 \%$ of those who reported that their jaw was locked or stuck once a week or more did not receive a related diagnosis according to the DC/TMD. This could partly be explained by the fact that the diagnostic algorithms related to TMJ intra-articular disorders, except disc displacement without reduction with limited opening, lack precision and are regarded more suitable for screening purposes than diagnostics (22). Nevertheless, the third question detected more severe TMJ dysfunction conditions without inclusion of more trivial conditions from the TMJ.

The present study is, to our knowledge, the first study to validate screening questions in relation to the DC/TMD criteria. A previously published screening instrument for TMD pain (12), when validated in relation to the RDC/TMD criteria, showed higher sensitivity as compared to the $3 \mathrm{Q} / \mathrm{TMD}$ when validated in relation to the DC/TMD criteria. There may be several reasons for this difference. One major difference is the choice of study population. The former study tested sensitivity and specificity of the screening questions on different subgroups already diagnosed with TMD or cleared of any such diagnosis. We examined positive and negative responders to the screening 3Q/ TMD in relation to a DC/TMD diagnosis in a clinical context, without previous exclusion of cases in the grey zone or with dental pain conditions. Some interesting similarities between the screening tests may be noted; both focus on pain on function as well as on pain of sufficient frequency during a recent period. One notable difference is that $3 \mathrm{Q} / \mathrm{TMD}$ includes one question on functional limitation.

For some individuals, the answers to the $3 \mathrm{Q} / \mathrm{TMD}$ differed between the first appointment at randomisation and the second appointment at the clinical research examination. We expected some fluctuations as there was a time gap ranging from three to 40 weeks between the two appointments, and it is well known that TMD symptoms tend to fluctuate over time (23). For practical reasons, it was not possible to recruit and examine the randomly selected individuals more closely in time to their regular dental check-up. The 3Q-positives and 3Q-negatives were defined based on answers to the 3Q/TMD at the second examination in order for any fluctuations not to influence the outcome of the study.

Of those who shifted from 3Q-positives to 3Qnegatives at the second examination, 14 received a DC/TMD diagnosis. Although this crossover between 3Q-positives and 3Q-negatives, and vice versa, could be regarded as a limitation of the present study design, it supports the view of fluctuating symptoms among individuals with musculoskeletal disorders (24). However, as all participants answered the screening questions again at the time of the clinical examination, we ensured that any possible crossover effects did not affect the results. In the present study, patients in the Public dental health were invited by letter after their routine dental check-up. Obviously, not all individuals invited to take part in a research study will accept to participate, but there was no difference between 3Q-positives and 3Q-negatives in the proportion of individuals who accepted the invitation.

The present study was based on an adult general population within the Public Dental Health Service in Västerbotten, Sweden, and is one of the first studies to evaluate screening questions used in a large population (10) in relation to the revised DC/TMD criteria. These criteria have been shown to be both valid and reliable for TMD pain. As all examinations were performed by a single and calibrated examiner trained to achieve a high reliability (Level 3) in the DC/TMD diagnostic system, the internal validity was assumed high. We consider the results valid for the adult population in Sweden and comparable countries.

In clinical practice, it is desirable to employ methods that effectively recognise patients with conditions that may require treatment. This should also involve a routine at regular dental check-ups to identify patients with more severe TMD. This is important since TMD is not only a condition which in itself will affect an individual's quality of life, but can also be involved in the development of other pain conditions $(25,26)$. Cost-effective treatments are available for 
TMD conditions, and early treatment efforts may prevent an escalation of the symptoms.

In conclusion, we consider that the $3 \mathrm{Q} / \mathrm{TMD}$ is a simple, applicable, cost-effective and valid tool for screening in a general adult population in order to recognise patients in need of further TMD examination and management.

\section{Acknowledgments}

The study was approved by the Ethical Review Board (Ref no 2012-331-31M, Umeå University, Sweden). Financial support was provided through regional agreement between Umeå University and Västerbotten County Council on cooperation in the field of Medicine, Odontology and Health, Swedish Dental Society and the Kempe Research Foundation. Dr. Lobbezoo reports Grants from Sunstar Suisse, grants from SomnoMed, outside the submitted work and Member of Academic Advisory Board (AAB) GrindCare - Sunstar Suisse.

\section{Disclosures}

The other authors have stated explicitly that there are no conflicts of interest in connection with this article.

\section{References}

1. Okeson JP. Management of temporomandibular disorders and occlusion. 7 ed. Elsevier: Mosby; 2012.

2. LeResche L. Epidemiology of temporomandibular disorders: implications for the investigation of etiologic factors. Crit Rev Oral Biol Med. 1997;8:291-305.

3. Fillingim RB, Slade GD, Diatchenko L, Dubner R, Greenspan JD, Knott C et al. Summary of findings from the OPPERA baseline case-control study: implications and future directions. J Pain. 2011;12(Suppl):T102-T107.

4. Maixner W, Diatchenko L, Dubner R, Fillingim RB, Greenspan JD, Knott C et al. Orofacial pain prospective evaluation and risk assessment study-the OPPERA study. J Pain. 2011;12(Suppl):T4-T11 el-2.

5. Manfredini D, Guarda-Nardini L, Winocur E, Piccotti F, Ahlberg J, Lobbezoo F. Research diagnostic criteria for temporomandibular disorders: a systematic review of axis I epidemiologic findings. Oral Surg Oral Med Oral Pathol Oral Radiol Endod. 2011;112:453-462.

6. Dahlström L, Carlsson GE. Temporomandibular disorders and oral health-related quality of life. A systematic review. Acta Odontol Scand. 2010;68:80-85.

7. Wänman A, Wigren L. Need and demand for dental treatment. A comparison between an evaluation based on an epidemiologic study of 35-, 50-, and 65-year-olds and performed dental treatment of matched age groups. Acta Odontol Scand. 1995;53:318-324.

8. Al-Jundi MA, John MT, Setz JM, Szentpetery A, Kuss O. Meta-analysis of treatment need for temporomandibular disorders in adult nonpatients. J Orofac Pain. 2008;22:97-107.

9. Swedish National Board of Health and Welfare, 2013. Available at: http://www.socialstyrelsen.se/nationalguidelines/ nationalguidelinesforadultdentalcare, Updated: 2013 , accessed 16 July 2016.

10. Lövgren A, Häggman-Henrikson B, Visscher CM, Lobbezoo F, Marklund S, Wänman A. Temporomandibular pain and jaw dysfunction at different ages covering the lifespan - a population based study. Eur J Pain. 2016;20:532-540.

11. Nilsson IM, List T, Drangsholt M. The reliability and validity of self-reported temporomandibular disorder pain in adolescents. J Orofac Pain. 2006;20:138-144.

12. Gonzalez YM, Schiffman E, Gordon SM, Seago B, Truelove EL, Slade G et al. Development of a brief and effective temporomandibular disorder pain screening questionnaire: reliability and validity. J Am Dent Assoc. 201 1;142:1183-1191.

13. Schiffman E, Ohrbach R, Truelove E, Look J, Anderson G, Goulet JP et al. Diagnostic criteria for temporomandibular disorders (DC/TMD) for clinical and research applications: recommendations of the international RDC/ TMD Consortium Network* and Orofacial Pain Special Interest Groupdagger. J Oral Facial Pain Headache. 2014;28:6-27.

14. Vilanova LS, Garcia RC, List T, Alstergren P. Diagnostic criteria for temporomandibular disorders: self-instruction or formal training and calibration? J Headache Pain. 2015; 16:505.

15. Ohrbach R, Larsson P, List T. The jaw functional limitation scale: development, reliability, and validity of 8 -item and 20-item versions. J Orofac Pain. 2008;22:219-230.

16. Von Korff M, Ormel J, Keefe FJ, Dworkin SF. Grading the severity of chronic pain. Pain. 1992;50:133-149.

17. Von Korff M, Dworkin SF, Le Resche L, Kruger A. An epidemiologic comparison of pain complaints. Pain. 1988;32:173-183.

18. Newcombe RG. Two-sided confidence intervals for the single proportion: comparison of seven methods. Stat Med. 1998; 17:857-872.

19. Dworkin SF, LeResche L. Research diagnostic criteria for temporomandibular disorders: review, criteria, examinations and specifications, critique. J Craniomandib Disord. 1992;6:301-355.

20. Wahlund $\mathrm{K}$, List T, Dworkin SF. Temporomandibular disorders in children and adolescents: reliability of a questionnaire, clinical examination, and diagnosis. J Orofac Pain. 1998; 12:42-51.

21. Nydell A, Helkimo M, Koch G. Craniomandibular disorders in children-a critical review of the literature. Swed Dent J. 1994; 18:191-205.

22. Michelotti A, Alstergren P, Goulet JP, Lobbezoo F, Ohrbach $\mathrm{R}$, Peck $\mathrm{C}$ et al. Next steps in development of the diagnostic criteria for temporomandibular disorders (DC/TMD): 
recommendations from the International RDC/TMD Consortium Network workshop. J Oral Rehabil. 2016;43: 453-467.

23. Marklund S, Wänman A. Risk factors associated with incidence and persistence of signs and symptoms of temporomandibular disorders. Acta Odontol Scand. 2010;68: 289-299.

24. Marklund S, Wänman A. Incidence and prevalence of myofascial pain in the jaw-face region. A one-year prospective study on dental students. Acta Odontol Scand. 2008;66:113-121.
25. Vierck CJ Jr. Mechanisms underlying development of spatially distributed chronic pain (fibromyalgia). Pain. 2006; 124:242-263.

26. Marklund S, Wiesinger B, Wänman A. Reciprocal influence on the incidence of symptoms in trigeminally and spinally innervated areas. Eur J Pain. 2010;14:366-371.

Correspondence: Anna Lövgren, Department of Clinical Oral Physiology, Faculty of Medicine, University of Umeå, Umeå 90187 , Sweden.

E-mail: anna.lovgren@umu.se 